\title{
Understanding Uneven Urban Expansion with Natural Cities using Open Data
}

\author{
YingLong $^{\mathrm{a}}$ WeixinZhai ${ }^{\mathrm{bc}}$ YaoShen $^{\mathrm{d}}$ XinyueYe $^{\mathrm{e}}$ \\ ${ }^{\text {a }}$ School of Architecture and Hang Lung Center for Real Estate, Tsinghua University, PR China \\ ${ }^{\mathrm{b}}$ Institute of Remote Sensing and Geographic Information System, Peking University, PR China \\ ${ }^{\mathrm{c}}$ Department of Geography and Earth Sciences, University of North Carolina at Charlotte, United States \\ ${ }^{\mathrm{d}}$ University College London, United Kingdom \\ e Kent State University, United States
}

\begin{abstract}
The last several decades have witnessed a rapid yet uneven urban expansion in developing countries. The existing studies rely heavily on official statistical yearbooks and remote sensing images. However, the former data sources have been criticized due to its non-objectivity and low quality, while the latter is labor and cost consuming in most cases. Recent efforts made by fractal analyses provide alternatives to scrutinize the corresponding "natural urban area". In our proposed framework, the dynamics of internal urban contexts is reflected in a quasi-real-time manner using emerging new data and the expansion is a fractal concept instead of an absolute one based on the conventional Euclidean method. We then evaluate the magnitude and pattern of natural cities and their expansion in size and space. It turns out that the spatial expansion rate of official cities (OCs) in our study area China has been largely under-estimated when compared with the results of natural cities (NCs). The perspective of NCs also provides a novel way to understanding the quality of urban expansion. We detail our analysis for the 23 urban agglomerations in China, especially paying more attention to the three most dominating urban agglomerations of China: Beijing-Tianjin-Hebei (BTH), Yangtze River Delta (YRD) and Pearl River Delta (PRD). The findings from the OC method are not consistent with the NC method, either. The distinctions may arise from the definition of a city, and the bottom-up NC method contributes to our comprehensive understanding of uneven urban expansion in the study area.
\end{abstract}

Keywords: Urban Expansion; Social Media; Head/Tail Division; New Data; China

\section{Introduction}

Most of urban expansion studies use remote sensing images or statistical yearbooks, and emerging new data provide new opportunities for understanding urban expansion. In this paper, we will examine national wide urban expansion using open data as well as referring to the idea of "Natural City" (NC) which means spatially clustered geographic events, such as the agglomerated patches aggregated from individual social media users' locations (Long, 2015; Jiang and Miao, 2015). Natural city may include natural town as well. NCs are a product of the bottom-up process in terms of data collection and geographic units or boundaries, which can be extracted from RS images, GPS and locationbased social media data (Jiang and Miao, 2015). Fractal geometry is the base of NCs. A fractal is a mathematical set that exhibits a repeating 
pattern which displays at every scale (Gouyet, 1996). Fractal geometry is a workable geometric middle ground between the excessive geometric order of Euclid and the geometric chaos of general mathematics. It is based on a form of symmetry that had previously been underused, namely invariance under contraction or dilation (Mandelbrot, 1983, Mandelbrot and Blumen, 1989). Researches on spatial analysis have concluded that artificially planned and designed spatial objects such as urban forms and transportation networks can also be treated as fractals (Batty, 2008, Clauset et al., 2009). The fractal structure of urban form is more evident when the urbanized areas of cities, metropolis, or urban systems are seen as a whole. Looking at urban growth/expansion in a fractal manner is an alternative perspective compared with the conventional Euclidean way (Batty, 1991, Chen, 2008, Chen, 2015). Thus, the overall form of the urbanized areas in a city can be treated as a fractal and effective measurements for spatial analysis. Not only does the introduction of fractal geometry provide a powerful tool for the mathematical description of a city system, but it also makes it possible for a number of geographic fractal simulations to compensate for the lack of geographic experimental studies (Batty and Longley,1986).

Despite the importance of urban expansion in developing countries like China, the rate and magnitude have not been universally validated in various sources. For instance, the statistical yearbooks are the main data sources for describing urban expansion for Chinese official cities (OCs), each of which has the explicit administrative boundary. Yearbooks have long been the basic channel for understanding urban expansion in China (Anderson and Ge, 2004, Li et al., 2015). Most of, if not all, existing urban studies for China adopt the administrative boundaries of OCs. The shortcomings of this dataset are summarized as the following. First, the existing OCs are not an objective way to observe the Chinese city system. Many towns have a large population, and they should be regarded as cities in the western context. However, they are not counted as the official city in China. In addition, some OCs are very rural. Second, the data quality of statistical yearbooks has been criticized for their resolution and informational richness. The geometrical properties and land use details of urban expansion are not recorded. Therefore, we cannot fully understand Chinese urban expansion using statistical yearbooks.

Besides the statistical yearbooks, remote sensing (RS) images are used to understand urbanization pattern and urban expansion. These RS images range from images with a resolution higher than $100 \mathrm{~m}$ by Landsat Thematic Mapper (TM), enhanced TM plus (ETM+) (Liu et al., 2005, Deng et al., 2010, Van de Voorde et al., 2011, Kuang et al., 2013) to the Operation Line-scan System (OLS) of the Defense Meteorological Satellite Program (DMSP) with 500m resolution (He et al., 2006, Liu et al., 2012). Due to its labor and cost consuming nature in some cases (Liu et al., 2003, Liu et al., 2005, Liu et al., 2010), remote sensing images cannot be easily applied to study urban expansion for all the cities in a timely fashion. In addition, RS analysis emphasizes the physical perspective of urban expansion without taking into account other dimensions. Therefore, an alternative approach to redefine the city system and understand its urban expansion from various dimensions is urgently needed to address the urban complexity.

Open Data have provided opportunities for quantitative urban studies (Reichman et al., 2011). Open data is the idea that some data should be freely available to everyone to use and republish as they wish, without restrictions from copyright, patents or other mechanisms of control (Auer et al., 2007). Our definition of open urban data is broad and refers to urban data that are openly accessible to the public, mainly come from three overlapping though different sources (Gurin et al., 2014): official data portals, big data initiatives, and the broader open data community. Ubiquitous open data from governments, commercial and social media websites are increasingly available to researchers. Open urban data have been a viable and cost-efficient option for understanding the built environment from both physical and social aspects, which has been particularly useful for the developing countries where data infrastructure is not sufficient (Long and Liu, 2015). The applications of open data 
have demonstrated their importance and suitability for urban studies, planning practice and commercial consultancy (Liu et al., 2016, Long and Liu, 2015).

In this study, we first redefine the city system by means of NCs using open data. We then derive urban expansion from various dimensions based on the derived NCs. In this paper, we initially distinguish urban expansion in two basic categories: size expansion and spatial expansion, corresponding to the conventional urban expansion using yearbooks and RS images, respectively. In this line, we are able to evaluate the identified size/pattern of urban expansion for various types of NCs for better understanding the process of urban growth. The identification and evaluation results for OCs can be benchmarked with those for NCs. Our proposed framework for understanding urban expansion with NCs is based on the fractal concept, within which the dynamics of internal urban contexts are reflected in a quasireal-time manner. The expansion is apart from an absolute one in the traditional method.

This paper is structured as follows: Section 2 introduces the main methods of the $\mathrm{NC}$ generation as well as the identification and characteristics of urban expansion. Section 3 presents the study area of and data used in the research. Section 4 outlines the results of urban area extraction, urban expansion identification and evaluation, along with benchmarking with other existing data sources. Section 5 summarizes academic contributions and indicates future work. Finally, Section 6 draws a conclusion.

\section{Methodology}

This paper adopts the analytical framework consisting of three modules depicted in Figure 1. The first module focuses on the identification of
NCs. The second concentrates on the evaluation and the shift in evolution of NCs on physical, morphological, functional and social dimensions for understanding urban expansion in size. The third module illustrates urban expansion in space, and evaluates its quality in terms of magnitude and pattern.

\subsection{Deriving natural cities from various dimensions (Module 1)}

A city is a relatively large human settlement according to the conventional definition. There is consistently a minimum population requirement. In this paper, we refer to Jiang and Liu (2012)'s approach to extract $\mathrm{NCs}^{*}$. It is based on the head/tail division rule stating given a variable $x$, if its values follow a heavy tailed distribution, the mean value of the values can divide all the values into two parts: a high percentage in the tail, and a low percentage in the head. The heavy tailed distribution refers to the statistical distributions that are right-skewed, for example, power law, lognormal, and exponential. In the previous studies, the densities of street nodes, the sizes of street blocks, and the nighttime imagery pixel values (DN), all demonstrated a heavy tailed distribution (Jiang, 2013, Jiang, 2015a, b, c). In this paper, the standard $\mathrm{NC}$ deriving procedures include four steps: 1) create a TIN with points (like road junctions); 2) convert TIN into TIN edges; 3) choose the edges less than the mean; 4) dissolve all the shorter edges into polygons to be individual NCs. This process is exemplified in Figure 2. Unlike the OCs defined by the administrative boundaries, NCs refer to human settlements or human activities in general on earth's surface that are objectively or naturally defined and delineated from massive geographic information. NC method is closer to the inherent structure of spatial entities, thus, providing us an alternative but valuable way to revisit the complex city system.

\footnotetext{
${ }^{*}$ We admit that some NCs may be greatly smaller than a city in common sense. These NCs as natural agglomerations may correspond to towns or streets in the
}

real world. In this paper, we use this term following Bin Jiang's definition (Jiang, 2015a, Jiang and Miao, 2015). 


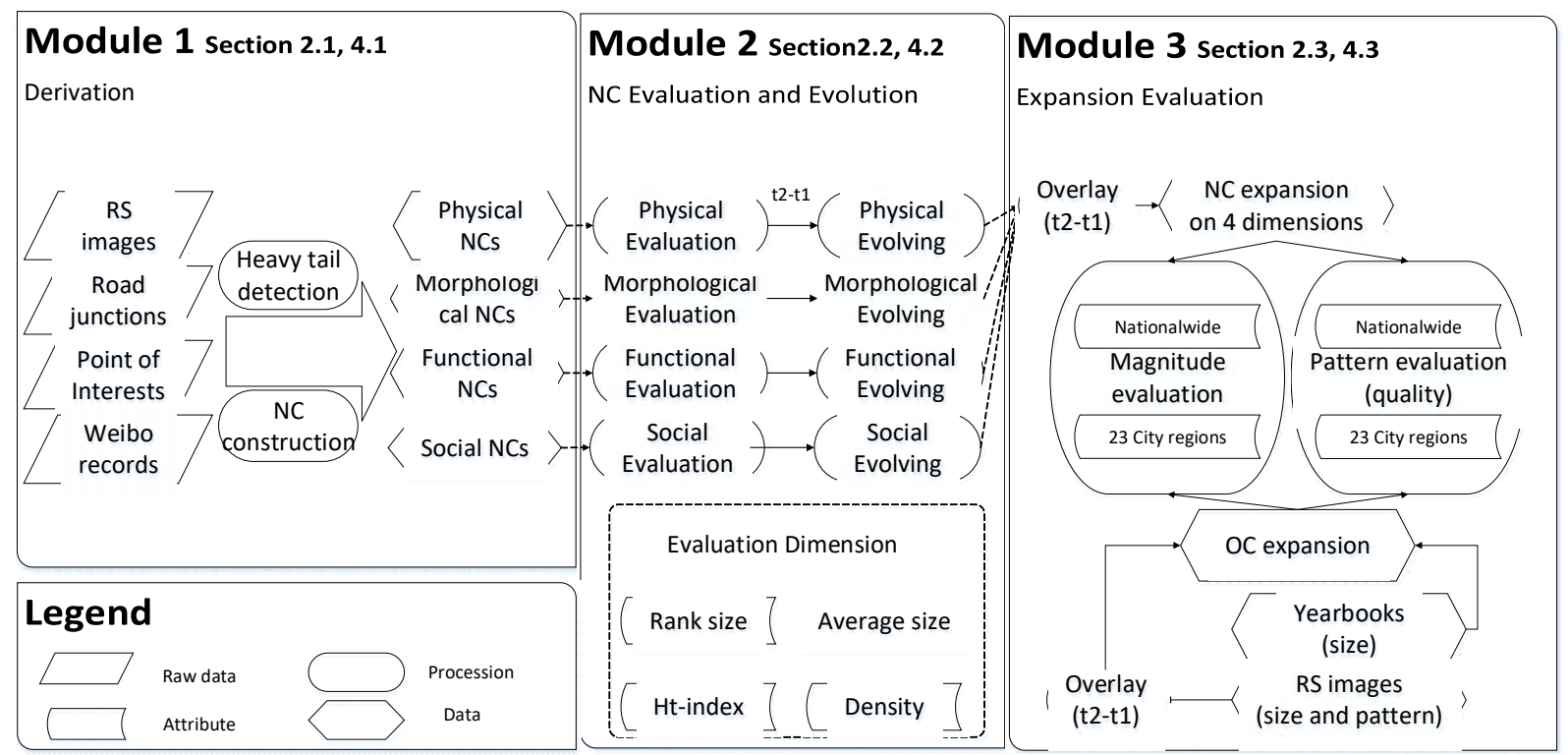

Figure 1. Overall framework of this study

Note: $\mathrm{t} 1=2009$ and $\mathrm{t} 2=2014$ in this paper.

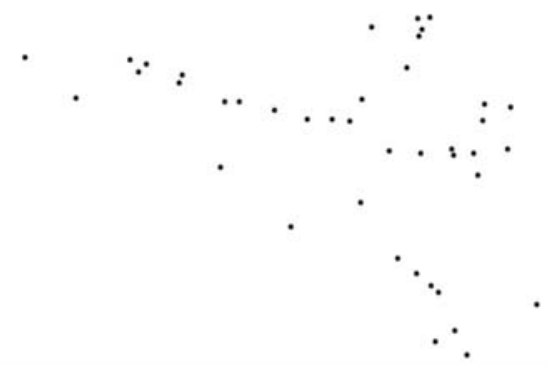

(a) Points

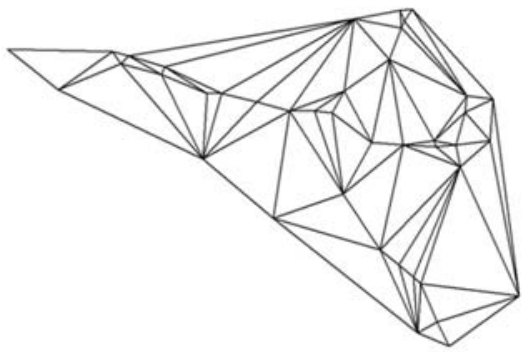

(c) Selected TIN edges

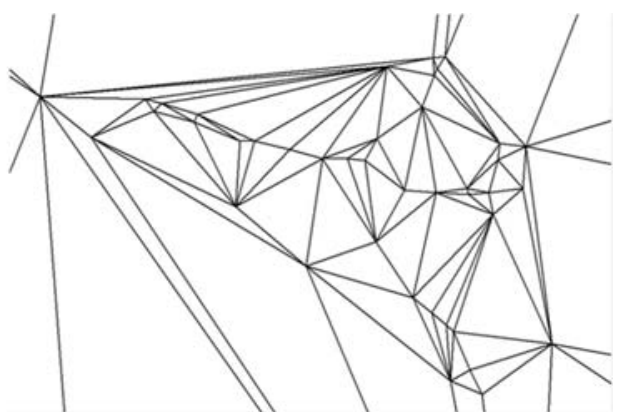

(b) TIN

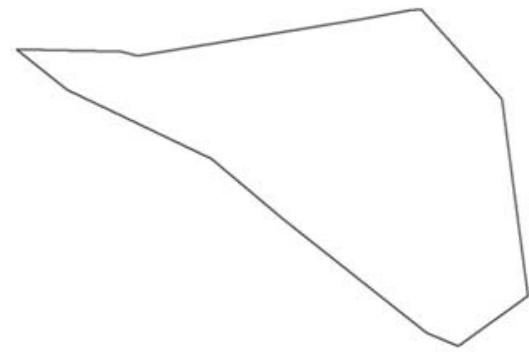

(d) Natural Cities

Figure 2. The four procedures for deriving natural cities from points 


\subsection{Evaluating evolving natural cities and their expansion in size (Module 2)}

Four types of NCs can be derived from multiple data sources, namely the physical NCs from remote sensing images, morphological NCs from road junctions, functional NCs from points of interest (POIs) and social NCs from social media records. The physical NCs extracted from the remote sensing images represent the skeleton of urban built-up areas. The road junctions are the elements of space connections in the built environment, thus making the corresponding $\mathrm{NCs}$ indicate the morphology of urban areas. The POIs generated NCs characterize the urban function distribution. The NC from social media records describe social significance of human activities. The physical-morphological-functional-social sequence is a path from physical developments to social developments, and represents a full chain of urbanization in space.

The evolution of NCs can be detected through deriving NCs during different periods. In so doing, five indicators are utilized to evaluate the NCs' evolution in size: rank size distribution, htindex, average city size, density, and expansion size. When we arrange all the cities according to their sizes, we get the rank-size plot. Considering the probability distribution of city sizes of all the derived NCs in China, we employ the head/tail division rule, a novel classification scheme for data with a heavy tailed distribution proposed by Jiang and his team (Jiang, 2013, Jiang and Yin, 2014, Jiang, 2015a). The ht-index measures the reoccurrence of the possible head/tail division, capturing the fractal properties of the NCs to describe the development degree (Jiang, 2015b, c). The head/tail division rule is superior to conventional classification methods in terms of capturing the inherent scaling pattern and the htindex of geographic feature $h$ exists if there are far more small geographic features than larger one at different scale when recursive times are h1 (Jiang, 2013, Jiang \& Yin, 2014). As for average size, that is literally. Density is the number of points (road junctions, POIs and social media records) per $\mathrm{km}^{2}$ within some specific urban area. A higher density represents more road junctions, POIs or social media records in the specific region, indicating the development/prosperity from the corresponding aspect. Last but not least, we define the expansion in size of NCs as the total urban area variation of NCs in a time period. For benchmarking with the expansion in size of the four types of NCs, we also derived the expansion in size for OCs using yearbooks or remote sensing images. Based on the comparison between OCs and NCs, we could gain knowledge from the definition of two different viewpoints of urban city.

\subsection{Evaluating urban expansion in space and its quality (Module 3)}

In addition to evaluating evolving NCs and their expansion in size, we can further explore more valuable meanings of urban expansion from the conventional way via focusing on the spatially expanded urban areas (urban expansion in space that are not urban in time $t_{1}$ and time $t_{2}$ ) both in $\mathrm{NCs}$ and OCs and compare the expanded areas with the originally existing urban areas to gain more knowledge on the quality of urban expansion in space. Different from the city evolution in module 2, city expansion in this module mainly concentrates on the city shape and the urban quality in different times. This can be achieved by using the overlay function in almost all GIS software, fed with urban areas from OCs or NCs in the aforementioned four dimensions.

Two indicators are proposed for evaluating urban expansion in space, magnitude and relative maturity. The magnitude of urban expansion in space is defined of the geometrical size of expanded urban areas. The ratio of magnitude and the urban area size in $t_{1}$ indicates the spatial urban expansion speed. The relative maturity indicator is used for measuring the quality of urban expansion in space in contrast to the originally existing urban areas, and is calculated as the ratio between the density of the newly expanded urban areas and that of the original urban areas in $t_{1}$. In this experiment, we measure the quality of expansion with the relative maturity indicator, which is calculated as the ratio between the density of the expanded urban areas and that of the urban areas in $t_{1}$. A higher quality represents a better development level of expansion, showing that there are a rapidly expansion increasing speed on some specific aspect. We suggest the 
ratio be calculated using road junctions for road junctions derived NCs, POIs for POIs derived NCs and social media records for social media records derived NCs. Road junctions, POIs and social media records are applied to the remote sensing images derived OCs.

\section{Data}

\subsection{The official cities in China and the observed expansion from yearbooks in 2009 and 2014}

The Chinese city system has a long history of being defined from the administrative view. Most of statistical data correspond to administrative Chinese cities (official cities, OCs), rather than concepts like metropolitan statistical area (MSA) in the United States or functional urban area (FUA) in European Union countries. There are totally 653 OCs in 2014 in China (Figure 3), with the sum of area of jurisdiction $782,330.9 \mathrm{~km}^{2}$. On the basis of the Chinese administrative system (Ministry of Housing and Urban Development, MOHURD, 2013, Marceau, 2005), there are mainly five categories of cities: municipalities directly leaded by the nation (MD, 4 cities), subprovincial cities (SPC, 15 cities), other provincial capital cities (OPCC, 17 cities), prefecture-level cities (PLC, 250 cities), and county-level cities (CLC, 367). The study area of this paper covers the whole Chinese territory, not limited to administrative areas of OCs in Figure 3 . However, the administrative boundaries are used to extract our analysis results in the whole country for benchmarking with the conventional indicators available in yearbooks.

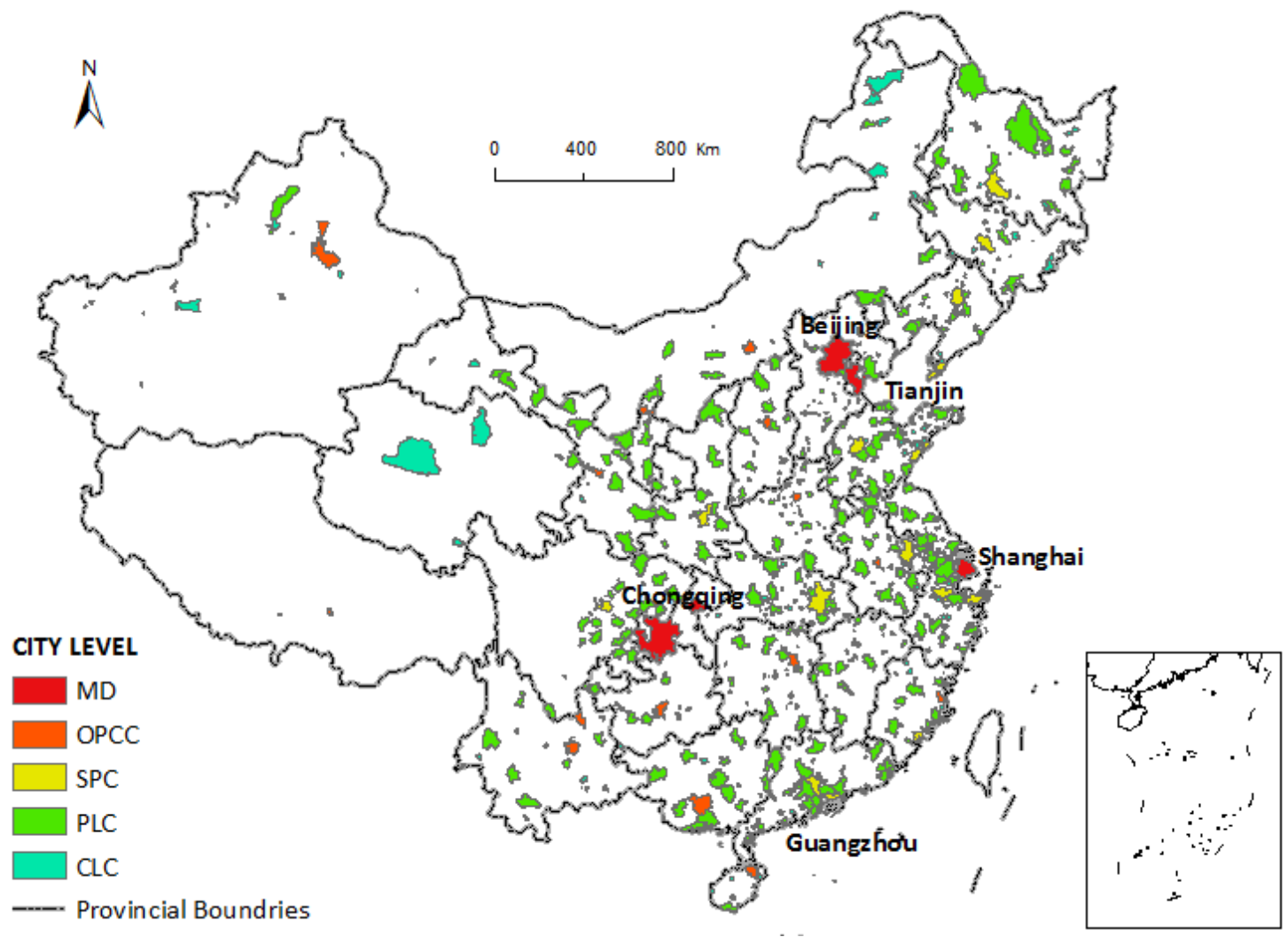

Figure 3. Administrative areas of all Chinese official cities

Note: Missing data in Taiwan 
China have been experiencing a rapid process of urbanization, with the urban residents occupying $17.9 \%$ of the nation's population in 1978 and 52.6\% in 2012 in China. As a result, China has become the world's largest and most rapidly transforming urban society (Bai et al., 2014; Wei and Ye, 2014a). People living in urban areas grew from $19.7 \%$ of the nation's population in 1978 to $49.7 \%$ in 2010 (Nation Bureau of Statistics, 1982, 2011). Besides, the uneven urban expansion rates are particularly salient in China, where annual rates of urban land expansion (1970-2000) vary from $13.3 \%$ in coastal areas to $3.9 \%$ in western regions (Seto et al., 2011). Due to rapid urbanization, large amounts of rural lands have been converted to built-up areas, and the irreversible and human dominated form of land use transformation has led to both environmental and socioeconomic problems. Urbanization in China has therefore attracted wide attentions from researchers, policy makers, investors, and the public. As Wei and Ye (2014b) stated, "an understanding of urbanization and land use change in China is required for appropriate strategies and policies to facilitate future sustainable development." For instance, the recently released "National New-Type Urbanization Plan (2014-2020)" has marked a new consensus among the country's ruling elites to facilitate sustainable and people-centered urbanization patterns (Bai et al., 2014, Wallace, 2014).

For gaining knowledge on the urban expansion of OCs, we extract urban areas of Chinese OCs in 2009 and 2014 from the yearbook MOHURD (2014). In 2014, the total urban area (UA) for the 653 Chinese cities is $49904.1 \mathrm{~km}^{2}$. The urban expansion from 2009-2014 is plotted in Figure 4.

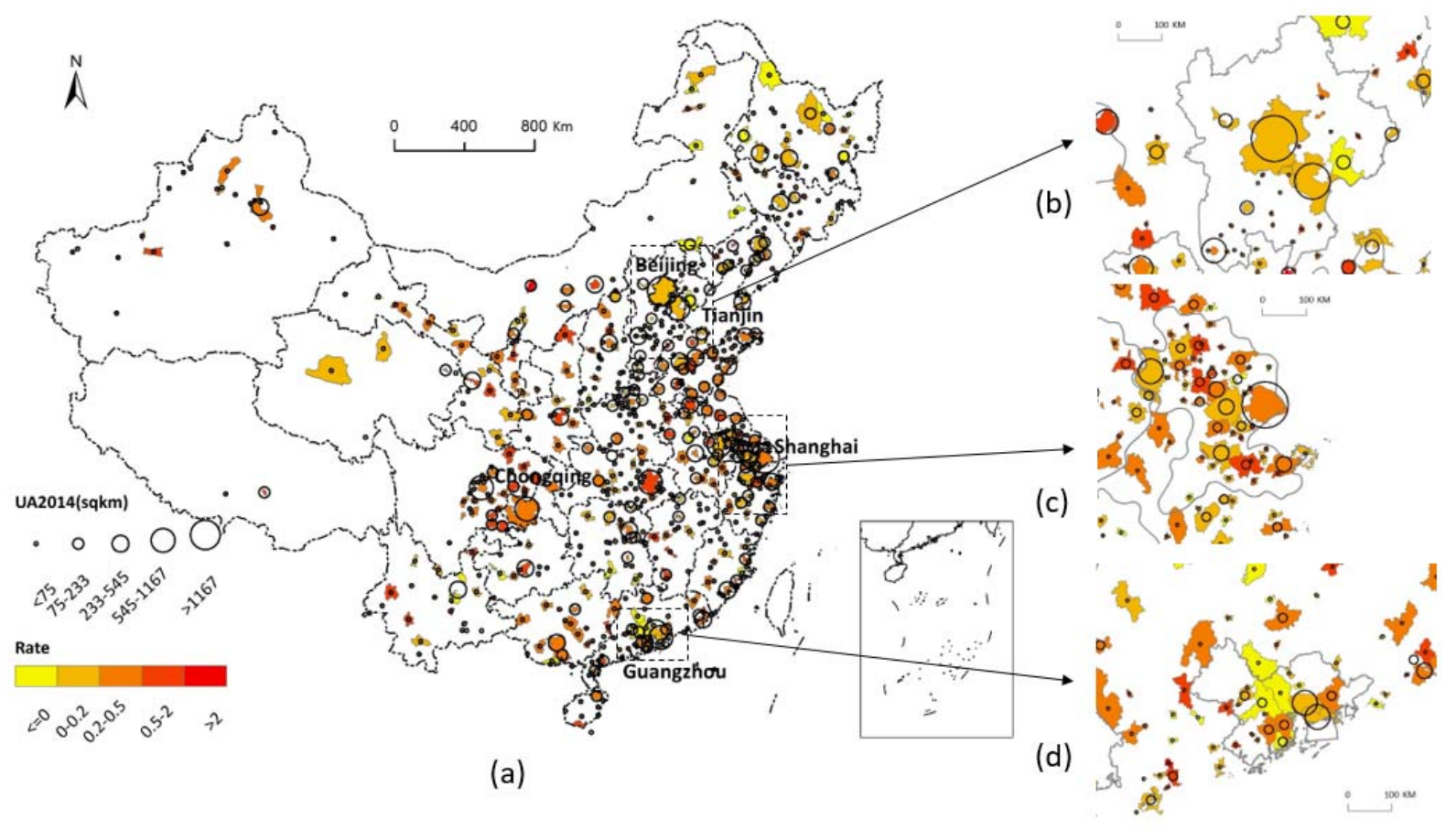

Figure 4. Urban areas in 2014 at the city level. (a) the whole China, (b) Beijing-Tianjin-Hebei (BTH), (c) Yangtze River Delta (YRD), (d) Pearl River Delta (PRD)

Note: The split thresholds in the process of head/tail division rules are chosen as the criteria for the legend classification. The rate denotes (UA2014-UA2009)/UA2009 for each city. Missing data in Taiwan. 
3.2 Road networks in ordnance surveys in 2009 and 2014-morphological data

We amassed the Chinese equivalent of Ordnance Survey data (hence the use of 'ORDNANCE' as the abbreviation) from China Siwei Surveying and Mapping Technology Co. Ltd. one of the largest navigation companies in China. Detailed road networks are available in the data. The total amount of all levels of road segments in 2009 and 2014 are 3,342,322 and 9,532,984, respectively. The pretreatment is conducted to connect nearby but topologically separated lines and the road junctions are generated from the road networks by using Network Analyst Tools in ESRI ArcGIS. There are 2,832,045 road junctions in 2009 and 9,054,366 road junctions in 2014, as shown in Figure 5(a,b).

\subsection{Points of Interest (POIs) in 2009 and 2014- functional data}

POIs in 2009 and 2014 are gathered and geocoded from business cataloguing websites (www.baidu.com). The initial twenty POI types are clustered into eight more general assemblies, among which commercial sites account for most POIs, followed by business establishments, transportation facilities, and government buildings. The data quality of POIs is secured through manual checking randomly selected POIs. There are 1,563,624 POIs in 2009 and 10,589,322 POIs in 2014, as shown in Figure $5(\mathrm{c}, \mathrm{d})$.

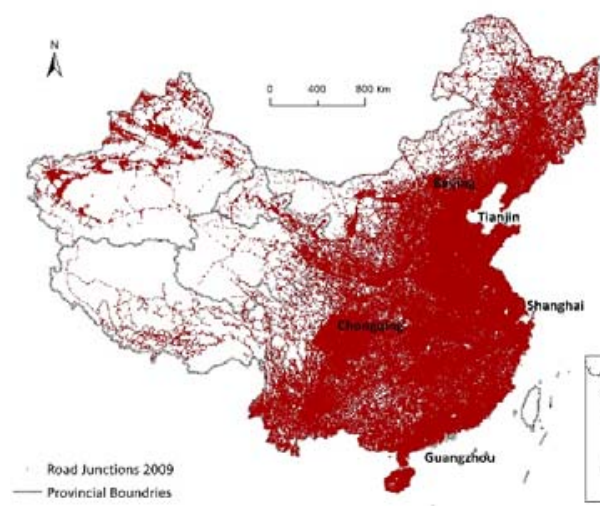

(a)

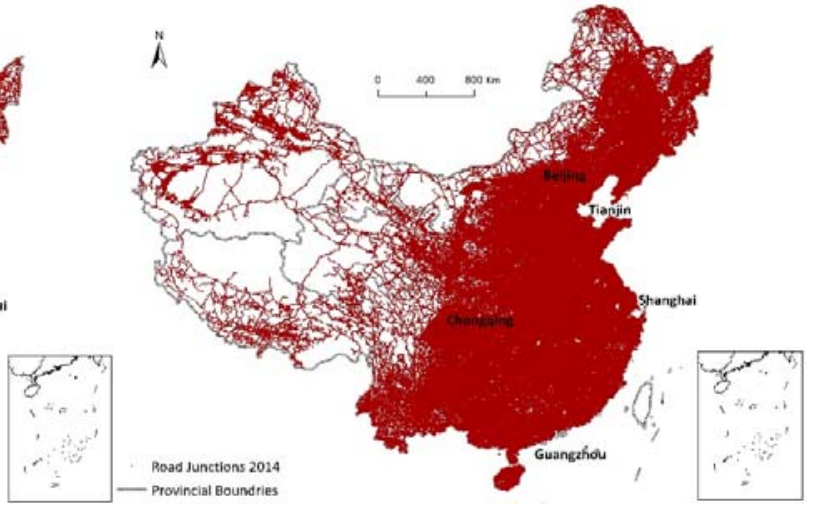

(b)

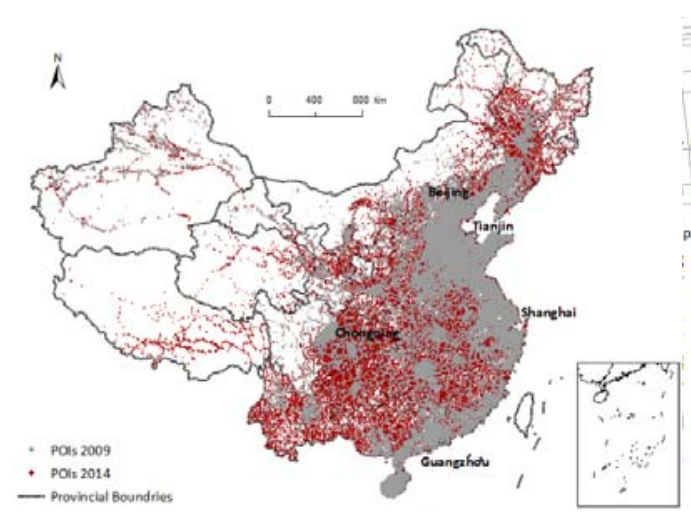

(c)

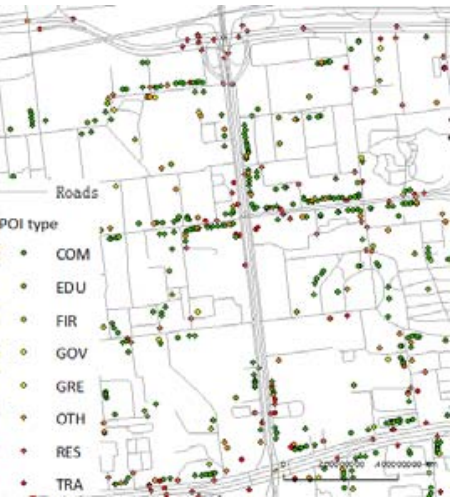

(d)

Figure 5. Road junctions and POIs of China in 2009 and 2014 (a) road junctions in the whole China in 2009, (b) road junctions in the whole China in 2014, (c) POIs in the whole China, (d) POIs in a part of the central city of Beijing Note: COM denotes commercial sites, EDU denotes education facilities, FIR denotes firms, GOV denotes government sites, GRE denotes green spaces, OTH denotes other types, RES denotes residence communities, and TRA denotes transport facilities. Missing data in Taiwan. 


\subsection{Urban areas interpreted from night light images in 2009 and 2014-physical data}

To derive NCs from the physical dimension, we gathered the urban areas presented in the $1 \mathrm{~km}$ resolution night light images retrieved from DMSP/OLS in 2009 (He et al., 2014) and in the $500 \mathrm{~m}$ resolution night light images retrieved from VIIRS/DNB (the updated version of DMSP/OLS) in 2014. The total urban areas in 2009 are $52,251.0 \mathrm{~km}^{2}$ with 2,166 patches (the mean patch size is $24.1 \mathrm{~km}^{2}$ ) for the whole China. We find that $26.4 \%$ of urban areas in 2009 was not urban in 2014, due to the different approaches adopted for image interpretation and the distinct spatial resolution of raw images. There are 12,752 urban patches with a total of $59,530.5 \mathrm{~km}^{2}$ in total in 2014, and the mean patch size is 4.7 $\mathrm{km}^{2}$.

\subsection{Weibo records in 2011 and 2014-social data}

Sina Weibo, the Chinese version of Twitter, is a leading online social media (microblogging) in China, which allows users to publish, share and discuss short messages on the website (http://www.weibo.com). Weibo is used by over $30 \%$ of Internet users in China, with a market penetration similar to Twitter. It was launched by SINA Corporation on 14 August 2009, and at the end of the fourth quarter of 2014, the number of monthly active users has reached 176 million, $80 \%$ of which come from mobile terminals. We collected the Weibo check-in records with geographical information in 2011 and 2014 (about 1\% Weibos have geo tags). There are 54,486 Weibo record points in 2011 and 874,542 Weibo records in 2014, along with the sharp increase in its user quantity. Due to incompleteness of data in Jiangxi and Hunan, our analysis on Weibo records does not cover the two provinces. In our research, we set the Weibo records in 2011 as the benchmark instead of 2009 in view of data availability.

\section{Results}

\subsection{Derived natural cities from various dimensions (Module 1)}

Following the method mentioned above, we established NCs based on remote sensing images, road junctions, POIs and Weibo records in the year 2009 and 2014, respectively (Weibo records in 2011 and 2014). The results are shown in Figure 6 . For better visualizing these NCs, we use the centroid to present an $\mathrm{NC}$ and apply the head/tail division rule for categorizing city sizes in area, and the threshold for the head/tail division is set at $40 \%$ in this study.

We are able to benchmark the two types of OCs and the four types of derived NCs of China from various dimensions, as revealed in Table 1 . The statistics of OCs from yearbooks and remote sensing images are similar due to stable urban development and the same total city amount. There are many more NCs than OCs in each year, which is evident to conform the arbitrariness of defining OCs. The total urban areas of the two types of physical and morphological NCs (RS images and road junctions) significantly increase from 2009-2014, while the functional NCs do not follow this trend. This indicates that the functional developments are far behind the physical and morphological developments of Chinese cities and might need more time to forester. The maximum NC size and standard deviation declined as well, indicating a tendency of homogenization and fragmentation. The point data in 2014 are relatively dense, leading to a lower value of cut distance in 2014 than in 2009. The ht-index is computed for all the patches as a whole, representing the development level of the entire country. The ht-index computed by RS images and road junctions hold unchanged, while that by POIs increases from 6 to 7 , and that by Weibo records increases from 4 to 6 . 


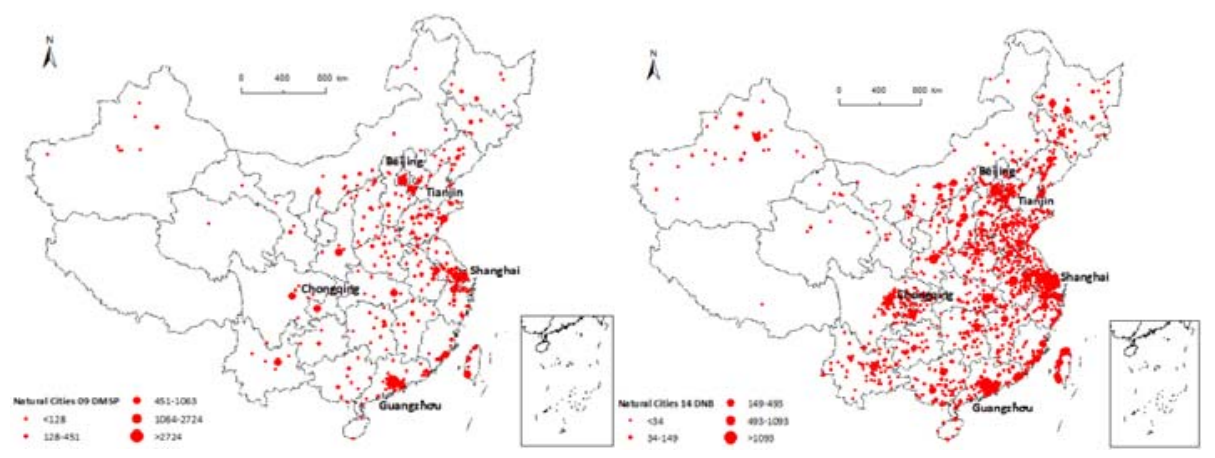

(a)

(b)

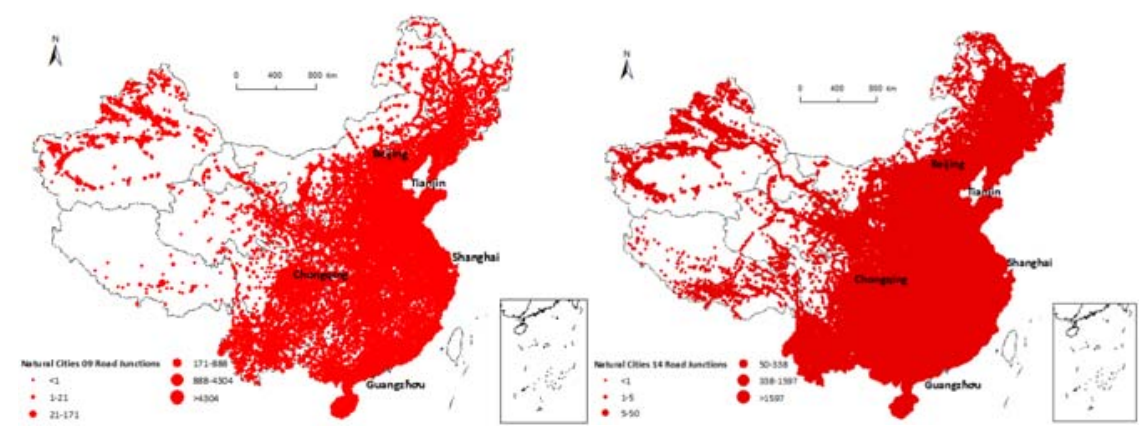

(c)

(d)

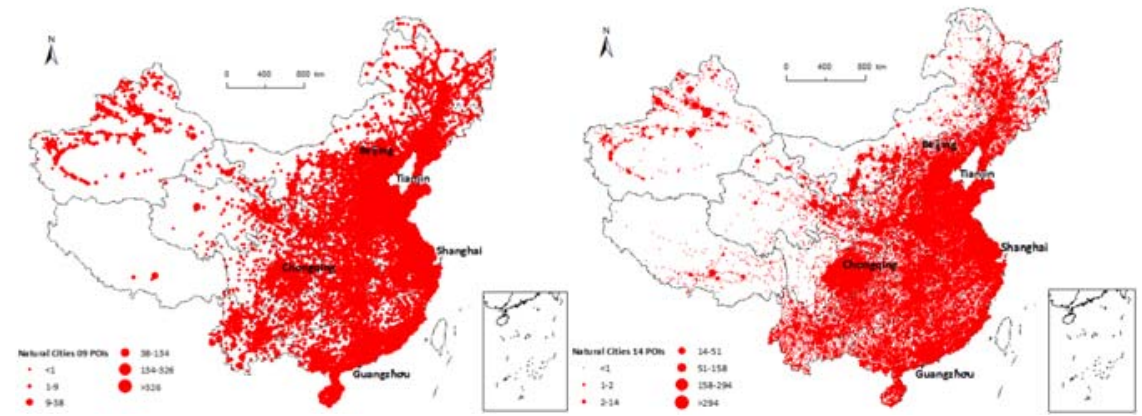

(e)

(f)

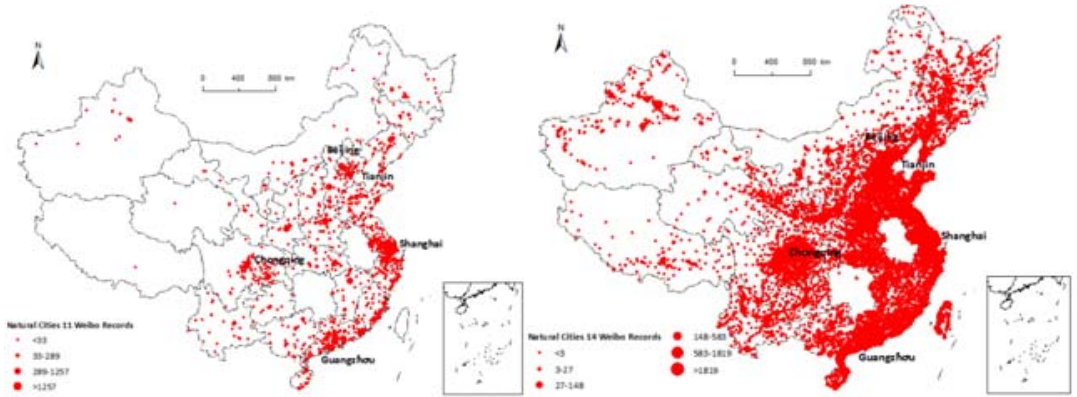

(g)

(h)

Figure 6. Four Types of Natural cities

Note: The NCs are derived from DMSP in 2009(a) and DNB in 2014(b), from road junctions in 2009(c) and 2014(d), from POIs in 2009(e) and 2014(f), and from Weibo records in 2011(g) and 2014(h) 
Table 1. Official cities (OCs) and derived natural cities (NCs)

\begin{tabular}{|c|c|c|c|c|c|c|c|}
\hline & $\begin{array}{l}\text { Average } \\
\text { city size } \\
\text { in } \mathbf{k m}^{2}\end{array}$ & $\begin{array}{l}\text { Maximum } \\
\text { size }\end{array}$ & $\begin{array}{l}\text { Total urban } \\
\text { area in } \mathbf{k m}^{2}\end{array}$ & $\begin{array}{l}\text { Standard } \\
\text { deviation } \\
\text { in } \mathbf{k m}^{2}\end{array}$ & \# cities & $\begin{array}{l}\text { Cut Distance } \\
\text { in meters }\end{array}$ & Ht-index \\
\hline $\begin{array}{ll}2009 & \text { OCs } \\
(\text { yearbooks) } & \end{array}$ & 63.3 & $2,429.1$ & $41,094.6$ & 140.2 & 649 & N/A & N/A \\
\hline $\begin{array}{ll}2014 & \text { OCs } \\
(\text { yearbooks }) & \end{array}$ & 75.7 & $2,915.6$ & $49,904.1$ & 168.0 & 659 & N/A & N/A \\
\hline $\begin{array}{ll}2009 & \text { OCs } \\
\text { (DMSP) } & \end{array}$ & 65.9 & $2,090.3$ & $43,401.5$ & 183.4 & 649 & $\mathrm{~N} / \mathrm{A}$ & N/A \\
\hline 2014 OCs (DNB) & 67.8 & $2,235.5$ & $44,707.4$ & 178.7 & 659 & $\mathrm{~N} / \mathrm{A}$ & N/A \\
\hline 2009 NCs (DMSP) & 128.8 & $4,725.4$ & $41,191.6$ & 320.7 & 326 & N/A & 5 \\
\hline 2014 NCs (DNB) & 34.5 & $3,995.2$ & $50,245.5$ & 142.1 & 1,458 & N/A & 5 \\
\hline $\begin{array}{l}2009 \text { NCs (road } \\
\text { junctions) }\end{array}$ & 1.6 & $8,439.1$ & $102,017.1$ & 57.0 & 65,707 & 880.8 & 6 \\
\hline $\begin{array}{l}2014 \text { NCs (road } \\
\text { junctions) }\end{array}$ & 0.4 & $11,396.3$ & $153,441.5$ & 29.5 & 347,886 & 587.0 & 6 \\
\hline 2009 NCs (POIs) & 1.0 & $1,263.6$ & $30,817.2$ & 13.2 & 29,674 & 963.0 & 6 \\
\hline 2014 NCs (POIs) & 0.2 & 724.4 & $29,332.8$ & 4.3 & 138,470 & 395.3 & 7 \\
\hline $\begin{array}{l}2011 \text { NCs (Weibo } \\
\text { records) }\end{array}$ & 33.6 & $9,712.8$ & $34,637.8$ & 327.9 & 1,031 & 4937.0 & 4 \\
\hline $\begin{array}{l}2014 \text { NCs (Weibo } \\
\text { records) }\end{array}$ & 3.2 & $6,743.8$ & $47,676.2$ & 62.6 & 14,741 & 1274.1 & 6 \\
\hline
\end{tabular}

Note: The cut distance is the length threshold used to select edges for constructing NC. For example, the cut distance of the 8,496,103 edges of NCs from road junctions in 2009 is $880.8 \mathrm{~m}$, which splits all the edges into two unbalanced parts: $1,679,315$ in the head (19.8\%) and 6,816,788 in the tail $(80.2 \%)$. The edges in the head part are used to build NCs. Because the RS images are not vector files, the data processing step is slightly different the other types of data sources. From the original RS images, we extract different patches according to the intensity of each pixel. Next, we select the patches which are larger than the average patch size as the natural cities. In this paper, $40 \%$ is chosen as the breakdown threshold. We also conducted an experiment with 50\% as threshold, and acquired similar results of Ht-index: 55666757 . 


\subsection{Evolving natural cities and their expansion in size (Module 2)}

We order the $\mathrm{NC}$ patches according to their sizes, and the rank-size plots of the four kinds of NCs are indicated in Figure 7. The sizes of NCs versus their ranks follow a heavy tailed distribution, suggesting that there are far more small patches than large ones. Two patterns can be observed from all distributions of NCs. The first is for the
NCs from large-scale datasets (road junctions, POIs and Weibo records), and the coefficients are around -2 . The second is for the NCs from RS images, and the coefficients are around -1 (Zip'f law featuring a coefficient of -1). The distributions at the tail part are also different for the two patterns, which may result from fewer city numbers in the RS images derived $\mathrm{NCs}$, compared to large-scale data derived NCs.

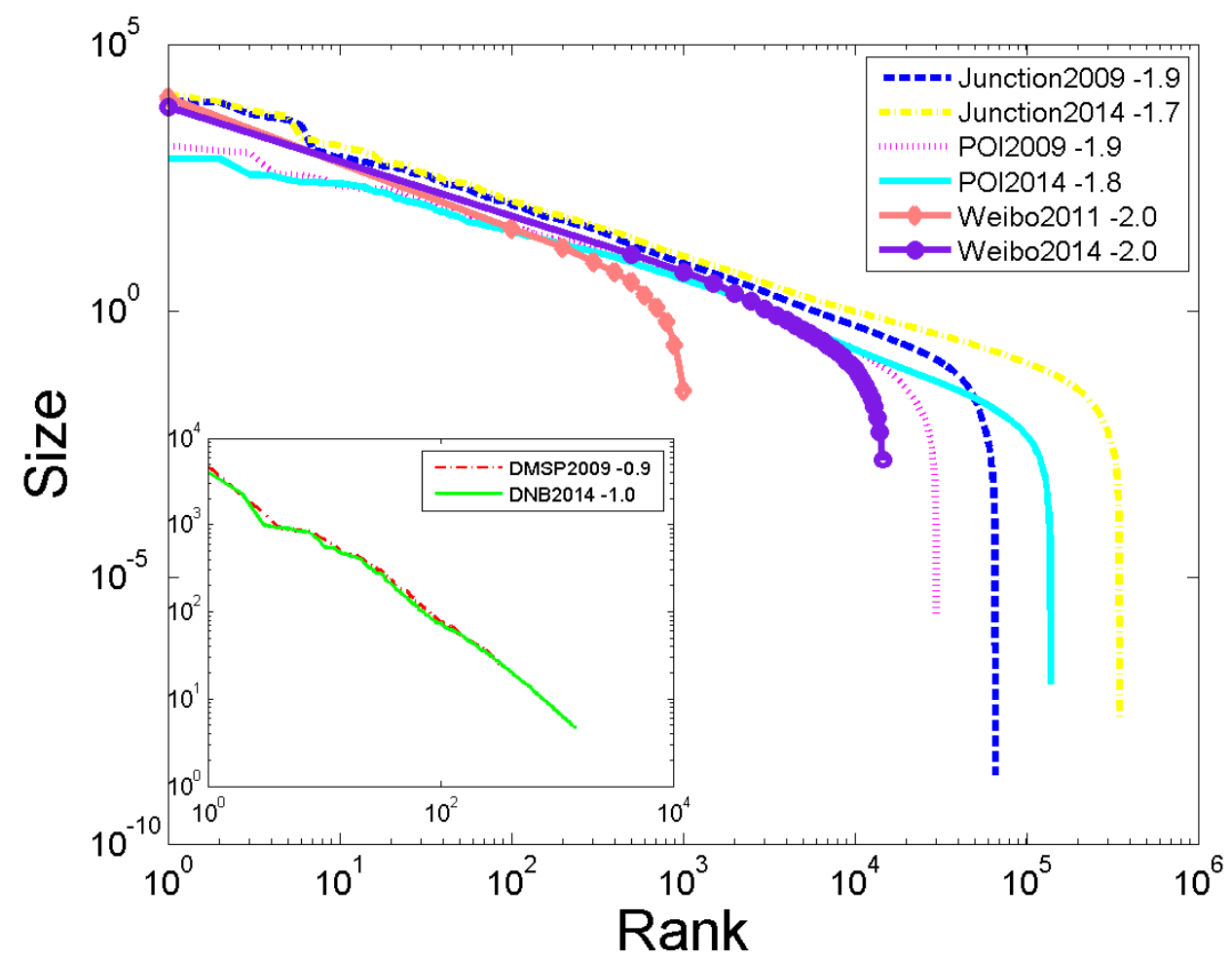

Figure 7. Rank-size distributions of four types of natural cities

Note: The coefficients of regressions between $\log ($ size $)$ and $\log ($ rank $)$ are indicated at the end of the legends.

We are able to gain knowledge on the urban expansion in size by comparing the features of the OCs and derived NCs. As indicated in Table 2, we find that the four types of NCs become compact in size from 2009-2014 from all four perspectives, as revealed by the average city size. The size expansion rate from 2009 to 2014 is $21.4 \%$ for the yearbook derived OCs and $3.0 \%$ for the RS images derived OCs, which may suggest that the official urban expansion speed has been underestimated by the yearbooks. However, the rate for $\mathrm{NCs}$ is $50.4 \%$ in the morphological dimension, $22.0 \%$ in the physical dimension, and
$-4.8 \%$ in the functional dimension, $37.6 \%$ in social dimension. In contrast to the official rate from the yearbooks, the transport infrastructure based urbanization is faster than the official rate, but the urban function developments are again confirmed to be behind the official rate and morphological rate.

We also evaluate NCs from the view of density, which is calculated as the number of points (road junctions, POIs and Weibo records) per $\mathrm{km}^{2}$ within NCs. This indicator represents the development prosperity in different aspects. The 
NCs in 2014 are associated with higher density than that in 2009, in both morphological and functional dimensions, indicating the morphological and functional developments are dramatically progressing within the NCs. In addition, the density of road junctions $\left(64.7 \mathrm{~km}^{-2}\right.$ and $\left.62.4 \mathrm{~km}^{-2}\right)$, POIs $\left(143.0 \mathrm{~km}^{-2}\right.$ and $\left.136.6 \mathrm{~km}^{-2}\right)$ and Weibo records $\left(12.6 \mathrm{~km}^{-2}\right.$ and $\left.11.9 \mathrm{~km}^{-2}\right)$ of OCs by remote sensing images are also shown in Table 2 for comparison. The RS images derived OCs are with higher density of road junctions and lower density of POI density.

Table 2. Descriptive information on evolving official cities (OCs) and natural cities (NCs)

\section{Official Cities $\quad$ Natural Cities}

\begin{tabular}{|c|c|c|c|c|c|c|}
\hline & Yearbooks & RS images & $\begin{array}{l}\mathrm{RS} \\
\text { images }\end{array}$ & $\begin{array}{l}\text { Road } \\
\text { junctions }\end{array}$ & POIs & $\begin{array}{l}\text { Weibo } \\
\text { records }\end{array}$ \\
\hline $\begin{array}{l}\text { Average city size in } \\
2009\left(\mathrm{~km}^{2}\right)\end{array}$ & 63.3 & 65.9 & 128.8 & 1.6 & 1.0 & 33.6 \\
\hline $\begin{array}{l}\text { Average city size in } \\
2014\left(\mathrm{~km}^{2}\right)\end{array}$ & 75.7 & 67.8 & 34.5 & 0.4 & 0.2 & 3.2 \\
\hline $\begin{array}{l}\text { Urban expansion in } \\
\text { size }\left(\mathrm{km}^{2}\right)\end{array}$ & $8,809.5$ & $1,305.9$ & $9,048.9$ & $51,424.4$ & $-1,484.3$ & $\begin{array}{l}13,038 . \\
4\end{array}$ \\
\hline $\begin{array}{l}\text { Size expansion rate } \\
(\%)\end{array}$ & 21.4 & 3.0 & 22.0 & 50.4 & -4.8 & 37.6 \\
\hline Density in $2009\left(\mathrm{~km}^{-2}\right)$ & N/A & $\begin{array}{l}64.7, \\
143.0,12.6\end{array}$ & $\mathrm{~N} / \mathrm{A}$ & 44.3 & 245.8 & 15.5 \\
\hline Density in $2014\left(\mathrm{~km}^{-2}\right)$ & N/A & $\begin{array}{l}62.4, \\
136.6,11.9\end{array}$ & $\mathrm{~N} / \mathrm{A}$ & 49.3 & 330.4 & 17.1 \\
\hline
\end{tabular}

Note: Urban expansion in size shows the difference between urban area sizes in 2014 and 2009. Size expansion rate is the ratio between increased urban areas and the urban areas in 2009. For Weibo records, the data are in 2011 and 2014.

\subsection{Urban expansion in space and its quality evaluation (Module 3)}

\subsubsection{The magnitude evaluation}

Urban expansion in space can be derived by employing the method in Section 2.3. The results from remote sensing images, road junctions, POIs and Weibo records shown in Table 3 reflect the physical, morphological, functional and social transformation. Different from urban expansion in size, the expanded urban areas generated from a spatial overlay operation are the areas that are non-urban in 2009 and urban in 2014 due to the adjustment of administrative boundaries. The spatial expansion rate is the ratio between expanded urban areas and urban areas of OCs or NCs in 2009. It is worth mentioning that there are some regions in NCs which existed in the year 2009 but disappeared in 2014. These areas are then not accounted for in urban expansion in space, but accounted for the size expansion calculation. We find that the spatial expansion rate of OCs has been largely underestimated when compared with the results of NCs. The four urban expansion rates in China are uneven, among which the one derived by road junctions is the most salient one, following by the one derived by Weibo records, much higher than that of functional NCs. This is consistent with the findings for urban expansion in size. 
Table 3. Urban expansion in space for OCs and NCs

\begin{tabular}{|c|c|c|c|c|c|c|}
\hline & \multicolumn{2}{|c|}{ Official Cities } & \multicolumn{4}{|c|}{ Natural Cities } \\
\hline & Yearbooks & $\begin{array}{l}\mathrm{RS} \\
\text { images }\end{array}$ & $\begin{array}{l}\mathrm{RS} \\
\text { images }\end{array}$ & $\begin{array}{l}\text { Road } \\
\text { junctions }\end{array}$ & POIs & $\begin{array}{l}\text { Weibo } \\
\text { records }\end{array}$ \\
\hline $\begin{array}{ll}\text { Expanded } & \text { urban } \\
\text { areas }\left(\mathbf{k m}^{2}\right) & \end{array}$ & $8,809.5$ & $11,869.5$ & $18,392.2$ & $71,363.1$ & $9,745.2$ & $21,459.0$ \\
\hline $\begin{array}{l}\text { Spatial expansion } \\
\text { rate }(\%)\end{array}$ & 21.4 & 27.4 & 43.8 & 70.0 & 31.6 & 62.0 \\
\hline
\end{tabular}

Note: We borrow the results of increased total urban areas and increased rate in Table 1 for the yearbooks derived OCs.

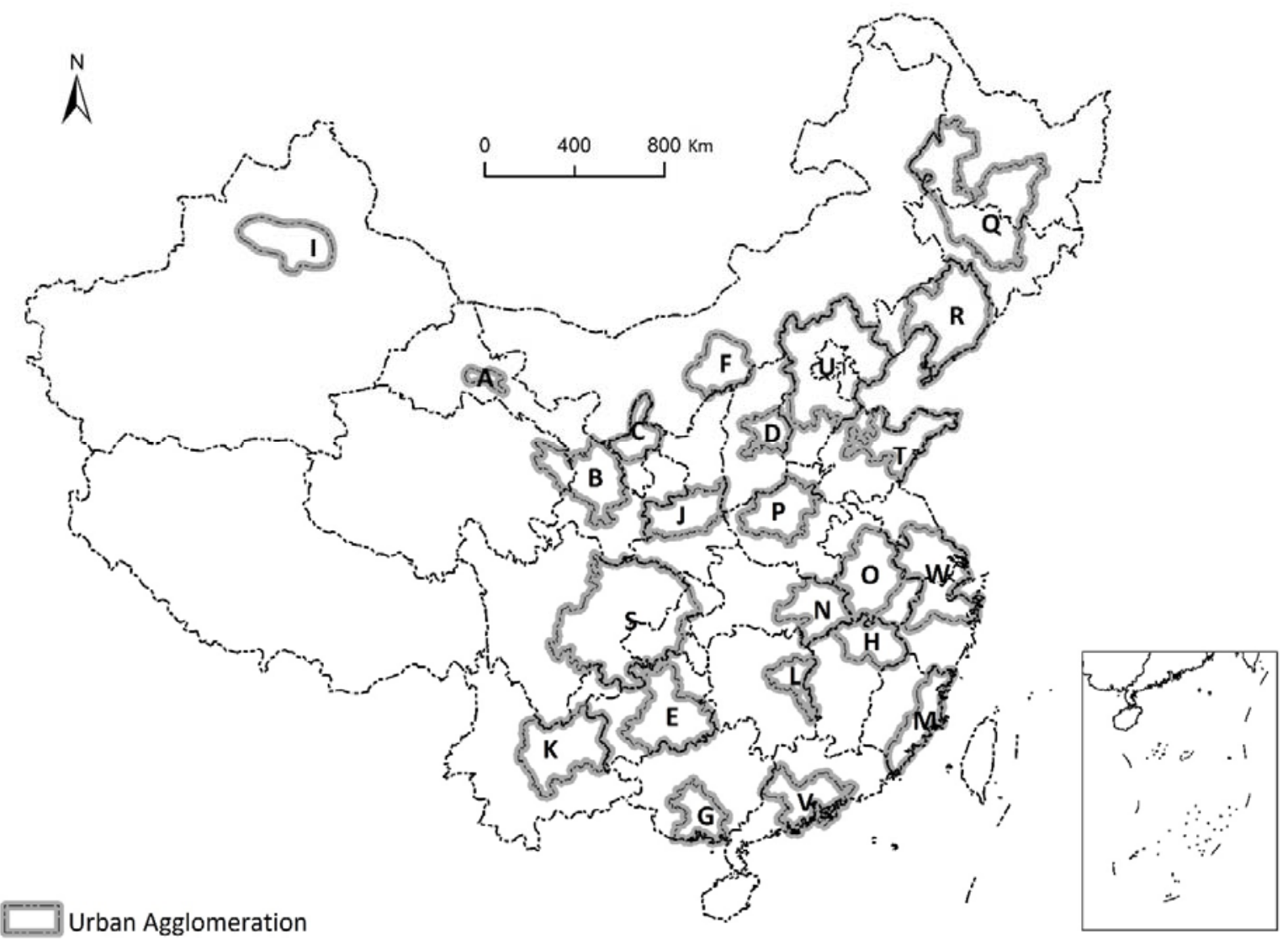

Figure 8. The 23 urban agglomerations in China

Note: The urban agglomerations are 1. Jiuquan-Jiayuguan-Yumen, 2. Lanzhou-Xining, 3. Northern Ningxia, 4. Central Shanxi, 5. Central Guizhou, 6. Central Inner Mongolia, 7. Southern Guangxi, 8. Northern Jiangxi, 9. Tianshan Mountains, 10. Guanzhong Plain, 11. Central Guizhou, 12. Eastern Hunan, 13. Eastern Fujian, 14. Eastern Hubei, 15. Central Anhui, 16. Central Plain, 17. Harbin-Changchun, 18. Liaodong Peninsula, 19. Chengdu-Chongqing, 20. Shandong Peninsula, 21. Beijing-Tianjin-Hebei, 22. Pearl River Delta, 23. Yangtze River Delta. 
For further investigation upon the urban expansion in space, we detail our analysis for the 23 urban agglomerations in China (Fand and $\mathrm{Yu}$, 2017, Long et al., 2014, Liu et al., 2015). These 23 urban agglomerations account for $21.3 \%$ of the total land resources, $55.7 \%$ of the total population, $64.2 \%$ of the total non-agriculture population, and $79.7 \%$ of the gross domestic product (GDP) in China in 2010 (Figure 8). The urban areas in 2009 and 2014, as well as the expanded urban areas of the 23 urban agglomerations are plotted in Figure 9, in which the urban agglomerations along the $\mathrm{x}$ axis follow the sequence 1-23 in Figure 8. As revealed by Figure 9, the urban expansion in space is a
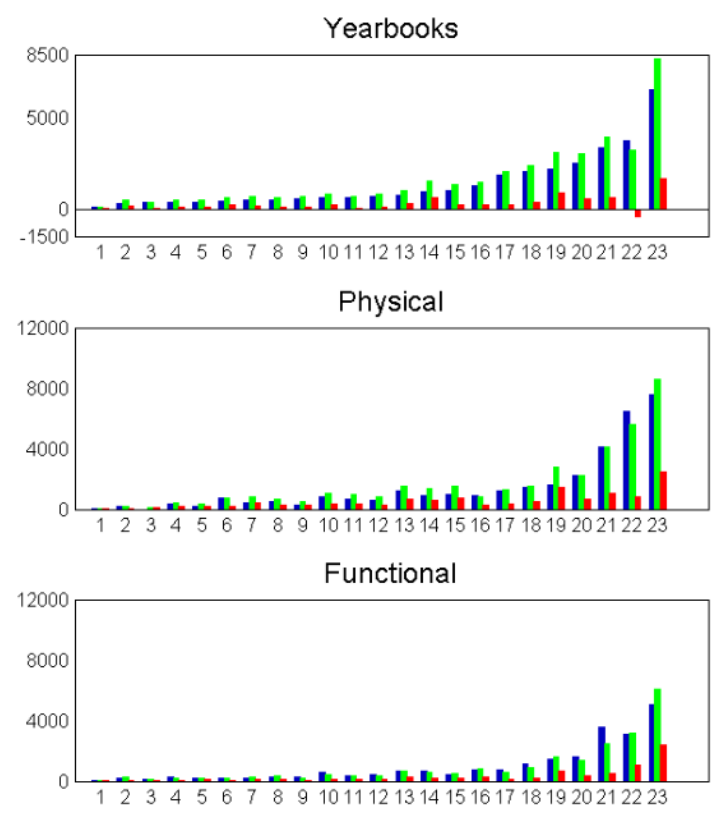

significant process across China in all the dimensions, both OCs and NCs. The rank of urban areas in 2014 in all dimensions is similar, indicating that the cities with larger urban areas in one dimension are often coming with larger areas in the other dimensions. In each dimension, there are great differences among the 23urban agglomerations, no matter regarding the urban areas in 2009 and 2014 or the urban expansion area. What is more, the variations among different dimensions within each urban agglomeration are still obvious: the morphological and social NCs form a sharp increasing from 2009 to 2014, while the functional NCs are not as remarkable.

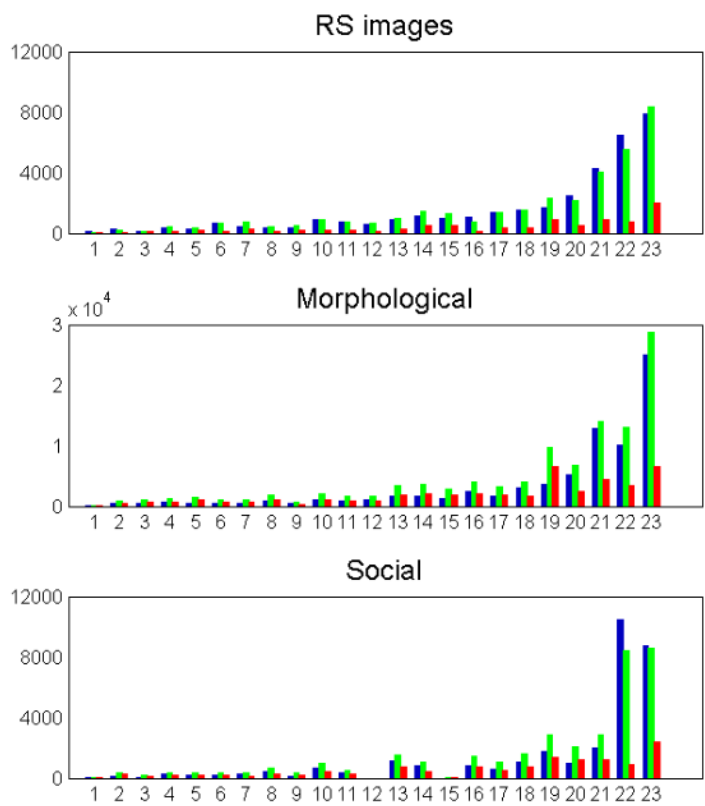

Figure 9. Magnitude evaluation of expanded urban areas of 23 urban agglomerations for OCs (the first two subfigures in the first row) and NCs (the other three subfigures) Note: The bars in blue, green and red represent the urban area sizes in 2009(2011), the urban area sizes in 2014 and the expanded urban area sizes. The $\mathrm{y}$-axis stands for the urban areas in $\mathrm{km}^{2}$. The expanded urban areas in the first subfigure is calculated as the increased urban areas in yearbooks from 2009-2014. Initially we used the same classes/ranges on the y axis on figure 9; while the 6 subfigures differ greatly and the universal classes/ranges make it awkward. Therefore, we do not use the same class/ranges on the y axis.

We pay more attention to the three most dominating urban agglomerations of China, Beijing-Tianjin-Hebei (BTH), Yangtze River Delta (YRD) and Pearl River Delta (PRD). The results are available in Figure 10. We notice significant pattern distinctions among all four types of NCs in 2009, as well as obvious differences among the four spatial expansion 
rates, revealing the mismatches among urban developments in various aspects. This provides a new lens for understanding urbanization, especially urban expansion, in China, in addition to the conventional aspect from yearbooks. There are also significant characteristics among the three typical urban agglomerations. In BTH, it expands greatly on the morphological and social dimension, while its functional development does not match. The land use policies and the rigid demand to working and living in Beijing primarily contribute to this irreversible expanding progress. Though Beijing and Tianjin have achieved a comparatively high urbanization level within a large scale, the expansion tendency is evidently intensive. Driven by the economic benefit, the local officials raise the revenue by transferring land use rights to developers, bringing about the substantially high housing prices and considerable expansion in Beijing. What is more, accounting for the transportation cost (monetary cost and time cost), the residents would prefer the land near the center as expected, and the center-outwards pattern is derived significantly. In YRD, the functional expansion rate is the largest percentage, indicating a considerable human-activity evolvement. As to the PRD, the NC from remote sensing images has the lowest expansion level, and the morphological and functional expansion form a coordinated development.

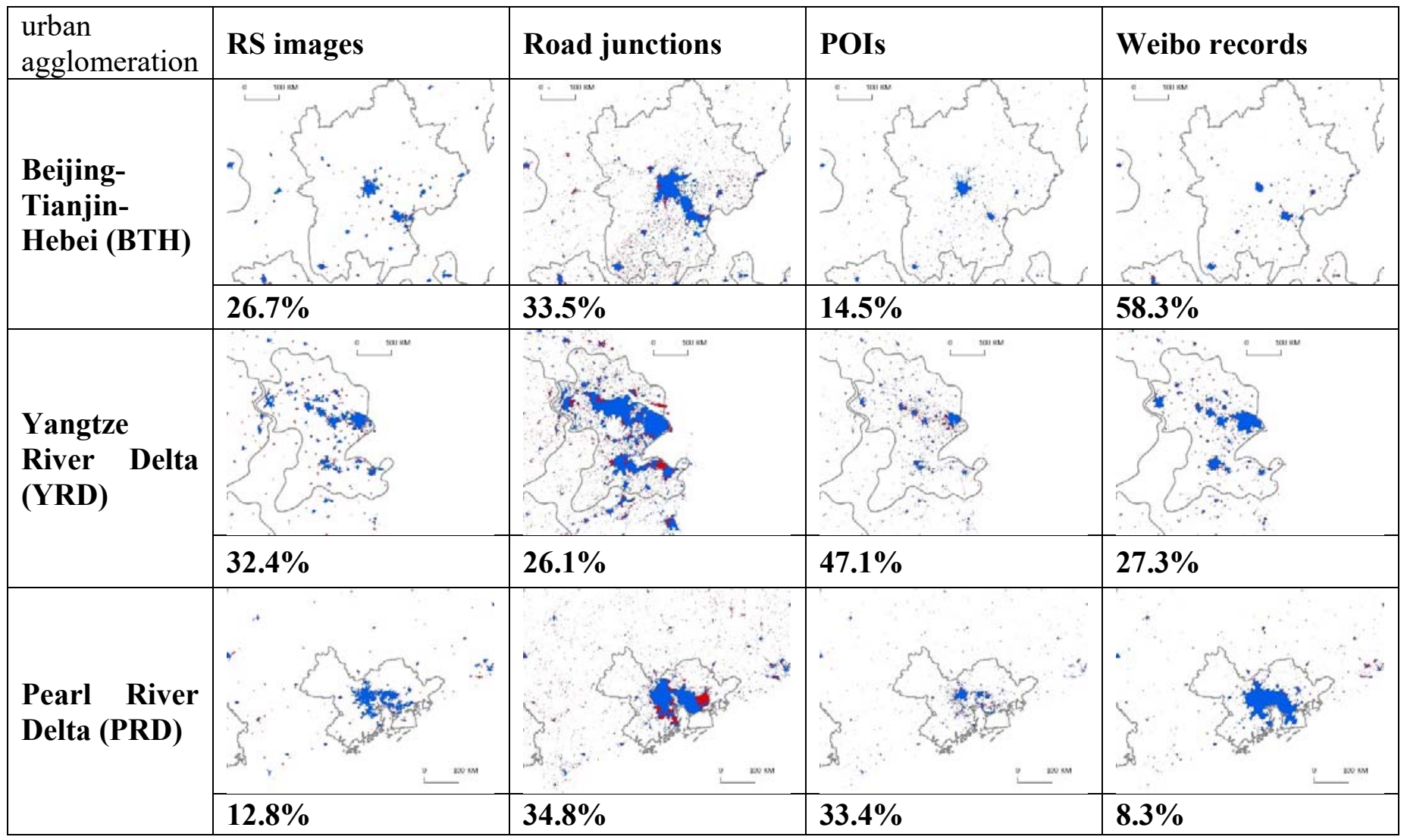

Figure 10. Urban expansion patterns of NCs in the three typical urban agglomerations

Note: The red denotes expanded urban areas from 2009-2014 and the blue denotes existing urban areas in 2009. The percentage under each subfigure represents the spatial expansion rate in a urban agglomeration. For Weibo records, the data are in 2011 and 2014. 


\subsubsection{The quality of urban expansion}

In addition to the magnitude evaluation on urban expansion in space, we measure the quality of expansion with the relative maturity indicator, which is calculated as the ratio between the density of the expanded urban areas and that of the urban areas in 2009, as shown in Table 4. The spatial expansion density of NCs is $42.5 \mathrm{~km}^{-2}$ and $39.1 \mathrm{~km}^{-2}$ for road junctions and POIs. The results for the remote sensing images derived OCs are calculated as well, and the density is much lower than that of NCs. In this regard, the quality of urban expansion from the view of NCs is good and approaching the quality of existing urban areas in 2009. However, the quality examination on urban expansion from the view of OCs indicates a very low level. This gap arises from the definition on a city e.g. a NC is generated based on the density of road junctions or POIs and those low density areas are excluded from the NC at the very derivation phrase. Nevertheless, the findings on the quality evaluation of Chinese urban expansion have provided a picture of understanding urban expansion from a new perspective.

Table 4. Quality evaluation of urban expansion in space

\begin{tabular}{|c|c|c|c|c|c|c|}
\hline \multirow[b]{2}{*}{ City Type } & \multicolumn{3}{|c|}{ Official Cities } & \multicolumn{3}{|c|}{ Natural Cities } \\
\hline & $\begin{array}{l}\text { Road } \\
\text { junctions }\end{array}$ & POIs & $\begin{array}{l}\text { Weibo } \\
\text { records }\end{array}$ & $\begin{array}{l}\text { Road } \\
\text { junctions }\end{array}$ & POIs & $\begin{array}{l}\text { Weibo } \\
\text { records }\end{array}$ \\
\hline Density of $2009\left(\mathrm{~km}^{-2}\right)$ & 64.7 & 143.0 & 12.6 & 44.3 & 245.8 & 15.5 \\
\hline $\begin{array}{l}\text { Spatial expansion } \\
\text { density }\left(\mathrm{km}^{-2}\right)\end{array}$ & 29.7 & 39.1 & 2.9 & 42.5 & 225.7 & 13.4 \\
\hline Relative maturity (\%) & 45.9 & 27.3 & 23.2 & 95.9 & 91.8 & 86.4 \\
\hline
\end{tabular}

Note: For Weibo records, the data are in 2011 and 2014.

We further examine the relative maturity of three typical urban agglomerations BTH, YRD and PRD for NCs and OCs, as indicated in Table 5. The relative maturity of $\mathrm{BTH}$ is $77.1 \%$ for morphological NCs and $140.1 \%$ for functional NCs. The conditions are different in the other two urban agglomerations. The morphological relative maturity indicators in YRD and PRD are $47.2 \%$ and $23.9 \%$, much lower than the national average. The functional relative maturity indicators in YRD and PRD are $65.1 \%$ and $63.5 \%$, also lower than the national average. The social relative maturities are consistent with the functional one, and for three regions they are $53.2 \%, 75.0 \%$ and $140.8 \%$. The low relative maturities on the three dimensions indicate two facts: Primarily, there are obvious distinctions between the highly developed central regions and the undeveloped marginal regions, especially within the large agglomerations. Secondly, the development of these urban agglomerations is approaching saturated; in other words, there seems to be a limitation for the urban expansion. The relative maturity examination for OCs is also scrutinized for benchmarking. The morphological relative maturity indicators of BTH, YRD and PRD are 39.2\%, 46.1\% and $62.6 \%$; while in functional dimension, they are $17.9 \%, 25.5 \%$ and $44.4 \%$ and $11.4 \%, 14.4 \%$ and $44.8 \%$ in social dimension. This finding from the $\mathrm{OC}$ view indicates that the quality of urban expansion in BTH is the worst, in contrast to PRD and YRD. The findings are not supported by the examination on the quality of urban expansion from the NC view, which again arises from the definition of a city. That is, some new developed areas of YRD are not regarded as urban areas in the derived NCs, due to low densities of both road junctions and POIs. 
Table 5. Relative maturity of three urban agglomerations

\begin{tabular}{lll|llll|}
\hline Urban & \multicolumn{3}{l}{ Official Cities } & \multicolumn{3}{l}{ Natural Cities } \\
\cline { 2 - 7 } agglomeration & morphological & functional & social & morphological & functional & social \\
BTH & $39.2 \%$ & $17.9 \%$ & $11.4 \%$ & $77.1 \%$ & $140.1 \%$ & $53.2 \%$ \\
YRD & $46.1 \%$ & $25.5 \%$ & $14.4 \%$ & $47.2 \%$ & $65.1 \%$ & $75.0 \%$ \\
PRD & $62.6 \%$ & $44.4 \%$ & $44.8 \%$ & $23.9 \%$ & $63.5 \%$ & $140.8 \%$ \\
\hline
\end{tabular}

\section{Discussion}

\subsection{Academic Contributions}

In this paper, we referred to the related academic work in the world to conduct an empirical study adopting NCs in China. This novel method comes from a completely fractal viewpoint and helps acquire the timely information concerning the dynamics, effects of urban expansion in China, and is also of the essence for policy making. The study contributes to existing urban expansion studies from the following aspects in detail. First, we apply the ideas of NCs to the case of urban expansion in China. The lightweight and straightforward methodology can facilitate timely urban expansion monitoring and evaluation for a large geographical area in the light of ubiquitous open data. The objectives and outcomes accurately reveal the differentiation of development, and prevent the influence of political and economic factors. The number of NCs from different open data varies, but it seems to have no obvious relation to the number of input data or the chosen cut distance. For example, the number of POIs in 2014 is almost ten times as large as that in 2009 and the cut distance shrinks to $40 \%$, while the total functional NC urban area decreases slightly, because of the urban functional agglomeration development mode. Second, we re-evaluate the urban system and five-year evolution of NCs which is well reflected in this study. As the fractal perspective is a different way from the traditional Euclidean method, the findings come from different viewpoints. The rank size, ht-index, average size, and density are considered as measurements to evaluate the complexity and integrity of all cities in China, thus providing an overall review on the redefined Chinese city system. The obtained NCs suggest that there is a quantity of regions with high-level development but not defined as a city in bureau. The increase of ht-index for NCs from POIs might suggest that functional expansion might need the support of its complexity, so the POIs are getting more and more clustered and complex with relatively lower degree of expansion. This is a total different expansion process. Third, we investigate the urban expansion magnitude and pattern in China. Road junctions represent the city's comprehensive transportation hub. Therefore, a large expansion rate of road junctions demonstrates a high degree of expansion on the morphological dimension. The POIs represent the urban functional developments, which obviously lag behind the morphological expansion in both space and quality. The social development is also obvious in both space and quality, this may arise from the phenomenon that Sina Weibo was lately created and the users of which proliferated. The results from $\mathrm{NC}$ method have proven the uneven urban expansions in China in different dimensions. The phenomena exist nationwide as well as locally (Jiang, 2015a, Jiang and Miao, 2015). The OC is a top-down definition from the government, which sometimes has policy guidance functions and usually fall lag the timely urban development. Therefore, the OC is supposed to be a biased and incomplete way of defining urban areas. In contrast, NC is a bottom-up method which is constructed on the basis of open data, reflecting the accurate urban city shape and urbanization progresses, and it is able to suggest regions which could not be identified by OC methods. Considering the requirements of cartography and also for simplicity, the OCs tend to omit some small patches of urban areas and aggregate the urban areas to be an integral entity, while the NCs artlessly demonstrate the natural urban forms; therefore, NCs outnumber the OCs in each year. The distinctions between NCs and OCs on the magnitude and quality evaluation with respect to the Chinese urban expansion may 
arise from the definition of a city, demonstrating that the proposed bottom-up NC method contributes to our comprehensive understanding on China's urban expansion from a new perspective.

\subsection{Potential bias and future steps}

This study focuses on the uneven urban expansion in China from an alternative perspective, and the adoption of emerging open data for natural city construction contributes to the study. Nevertheless, several limitations still exist in the current study which should be highlighted in our future research. First, NCs in 2014 do not entirely cover NCs of 2009, that is, there are several regions which are considered as natural cities in 2009 but not in 2014. This phenomenon is incompatible with the Chinese overall urbanization process which should be paid more attention. Second, the complicated urban expansion is supposed to be more than four aspects aforementioned in our research, and more open data sources are demanded to feed our proposed framework. Third, the inconsistency, incomparability of different open data sources may give rise to the disagreement or incongruity of the practical application in this proposed uniformed framework which is hard to avoid so far. Last but not least, unrepresentativeness and the occasional inaccessibility of the open data may contribute to the bias of the conclusion. More comprehensive data acquisition methods are supposed to reduce the inadequacy and impartiality to the utmost.

\section{Conclusions}

Research on China's urbanization has unfolded a complex landscape of spatial development. There has been a renewed interest in spatial inequality of urbanization, fueled by the concern over the effects of globalization and liberalization, and facilitated by theoretical and methodological developments in geography, economics, and computational social science. As Wei (2015) argues, "the popularity of social media provides new tools to study the nature of human behavior and spatial interaction. Social media has also produced new space of inequality, which has yet to be fully understood". This paper demonstrates such an implementation from open data perspectives including social media data.

In this paper, we have explored urban expansion in China based on the theory of natural cities (NCs), by using remote sensing images (physical dimension), road junctions (morphological dimension), POIs (functional dimension) and Weibo records (social dimension), respectively. In addition, the applications are tested in 2009 and 2014, enabling a lens on understanding urbanization especially the unevenness of urban expansion in China. To facilitate urban expansion from the view of NCs, we extended the concept of urban expansion to urban expansion in size and urban expansion in space. The findings on four dimensions of NCs are also benchmarked with yearbooks and remote sensing images derived from official cities (OCs) to demonstrate the applicability of our proposed framework. The research on Chinese cities heavily relied on statistical yearbooks and reports, prior to the emergence of open data. The aggregated information corresponds to administrative cities, rather at the intra-urban level. As we have indicated in the introduction section of the paper, the administrative cities in China are not perfect for understanding the Chinese city system, since some cities are not included in administrative cities and some large administrative cities are not real cities from the spatial entity dimension. The accuracy of the official information is also not always reliable. The open data used in this study do help in facilitating us understanding a more comprehensive and objective Chinese city system from various dimensions like morphology, function and activity. The open data make it possible to derive and evaluate $\mathrm{NCs}$, and characterize the magnitude and pattern of their temporal evolution. It can facilitate the next-step research on identifying distinct models of urban development and the significant role of driving forces.

The detailed findings of this paper are as follows. First, The NCs outnumber the OCs in each year, further confirming the setting problems of OCs. The total urban areas of the four types of NCs derived significantly increase from 2009-2014, especially for the morphological and social ones. Second, within each type of NC, we could 
observe a tendency of homogenization and fragmentation. The heavy tailed distribution for the sizes of NCs versus their ranks has been confirmed, indicating that there are far more small NCs than large ones. Third, while defining size expansion rate as the ratio between increased urban areas and the urban areas in 2009, we find that in contrast to the official rate from yearbooks, the transport infrastructure based urbanization in terms of road junction development is faster than the official rate, but the urban function developments are again confirmed to be behind the official rate and morphological rate. Fourth, given density for points (road junctions, POIs and Weibo records), the NCs form an increasing trend while the OCs decrease from 2009-2014. Fifth, the spatial expansion rate is set as the ratio between expanded urban areas and urban areas of OCs or NCs in 2009 for magnitude evaluation. Among the four expansion rates of NCs, the one derived by road junctions is the most significant, which is consistent with the urban expansion in size findings. The expansion derived by Weibo records is also noticeable in size and quality which may result from the sharp increase of Sina Weibo users from 2011 to 2014. Last, we measure the quality of expansion with the relative maturity indicator, which is calculated as the ratio between the density of expanded urban areas and that of the urban areas in 2009. We find a large gap between the relative maturity of OCs and NCs which may arise from the city definition.

\section{References}

Anderson, G. and Y. Ge (2004) Do economic reforms accelerate urban growth? The case of China. Urban Studies 41, 2197-210.

Auer S, Bizer C, Kobilarov G, et al. Dbpedia: A nucleus for a web of open data[M]//The semantic web. Springer Berlin Heidelberg, 2007: $722-735$.

Bai, X., P. Shi and Y. Liu (2014) Society:

Realizing China's urban dream. Nature 509, 158.
Batty M, Longley P A. The fractal simulation of urban structure[J]. Environment and Planning A, 1986, 18(9): 1143-1179.

Batty, M. (1991) Cities as fractals: simulating growth and form., Fractals and Chaos, Springer, 43-69.

Batty, M. (2008) The size, scale, and shape of cities. Science 319, 769-71.

Chen, Y. (2015) Power-law distributions based on exponential distributions: Latent scaling, spurious Zipf's law, and fractal rabbits. FractalsComplex Geometry Patterns and Scaling In Nature And Society, 1550009.

Chen, Y.G. (2008) Fractal urban systems: scaling, symmetry, and spatial complexity., Scientific Press, Beijing, China.

Clauset, A., C.R. Shalizi and M.E. Newman (2009) Power-law distributions in empirical data. Siam Review 51, 661-703.

Deng, X., J. Huang, S. Rozelle and E. Uchida (2010) Economic growth and the expansion of urban land in China. Urban Studies 47, 813-43.

Fang, C., and D. Yu (2017) Urban agglomeration: An evolving concept of an emerging phenomenon. Landscape and Urban Planning 162, 126-136.

Gouyet, Jean-François (1996). Physics and fractal structures. Paris/New York: Masson Springer.

Gurin, J. (2014). Open data now: The secret to hot startups, smart investing, savvy marketing, and fast innovation. New York, NY: McGrawHill.

He, C., P. Shi, J. Li, J. Chen, Y. Pan, J. Li, L. 
Zhuo and T. Ichinose (2006) Restoring urbanization process in China in the 1990s by using non-radiance-calibrated DMSP/OLS nighttime light imagery and statistical data. Chinese Science Bulletin 51, 1614-20.

He, C., Z. Liu, J. Tian and Q. Ma (2014) Urban expansion dynamics and natural habitat loss in China: a multiscale landscape perspective. Global Change Biology 20, 2886-902. Jiang, B. (2013) Head/tail breaks: A new classification scheme for data with a heavytailed distribution. The Professional Geographer 65, 482-94.

Jiang, B. (2015a) Head/tail breaks for visualization of city structure and dynamics.

Cities 43, 69-77.

Jiang, B. (2015b) Wholeness as a Hierarchical Graph to Capture the Nature of Space. International Journal of Geographical Information Science 29(9), 1632-48. Jiang, B. (2015c) A City Is a Complex Network. M. W. Mehaffy (Editor, 2015), Christopher Alexander. A City is Not a Tree: 50 th Anniversary Edition, Sustasis Press: Portland, $O R, x x-x x$.

Jiang, B. and J. Yin (2014) Ht-index for quantifying the fractal or scaling structure of geographic features. Annals of the Association of American Geographers 104, 530-40. Jiang, B. and X. Liu (2012) Scaling of geographic space from the perspective of city and field blocks and using volunteered geographic information. International Journal of Geographical Information Science 26, 215-29.
Jiang, B. and Y. Miao (2015) The evolution of natural cities from the perspective of locationbased social media. The Professional Geographer 67, 295-306.

Kuang, W., J. Liu, Z. Zhang, D. Lu and B. Xiang (2013) Spatiotemporal dynamics of impervious surface areas across China during the early 21 st century. Chinese Science Bulletin 58, 1691-701.

Li, H., Wei, Y., Liao, F. and Huang, Z. (2015) Administrative hierarchy and urban land expansion in transitional China. Applied Geography, 56, 177-186.

Liu, J., M. Liu, D. Zhuang, Z. Zhang and X. Deng (2003) Study on spatial pattern of land-use change in China during 1995-2000. Science in China Series D: Earth Sciences 46, 373-84. Liu, J., M. Liu, H. Tian, D. Zhuang, Z. Zhang, W. Zhang, X. Tang and X. Deng (2005) Spatial and temporal patterns of China's cropland during 1990-2000: an analysis based on Landsat TM data. Remote Sensing of Environment 98, 44256.

Liu, J., Z. Zhang, X. Xu, W. Kuang, W. Zhou, S. Zhang, R. Li, C. Yan, D. Yu and S. Wu (2010) Spatial patterns and driving forces of land use change in China during the early 21 st century. Journal of Geographical Sciences 20, 483-94. Liu, X., Derudder, B., \& Wu, K. (2016). Measuring polycentric urban development in China: an intercity transportation network perspective. Regional Studies, 50(8), 1302-1315. Liu, X., Y. Song, K. Wu, J. Wang, D. Li and Y. Long (2015) Understanding urban China with 
open data. Cities.

Liu, Z., C. He, Q. Zhang, Q. Huang and Y. Yang (2012) Extracting the dynamics of urban expansion in China using DMSP-OLS nighttime light data from 1992 to 2008. Landscape and Urban Planning 106, 62-72.

Long Y, Wu K, Mao Q. Simulating urban expansion in the parcel level for all Chinese cities[J]. arXiv preprint arXiv:1402.3718, 2014. Long, Y. and L. Liu (2015) Big/open data in Chinese urban studies and planning: A review. ISOCARP REVIEW 11.

Long, Y. (2016). Redefining Chinese city system with emerging new data. Applied Geography 75, 36-48.

Mandelbrot B B. The fractal geometry of nature[M]. Macmillan, 1983.

Mandelbrot B B, Blumen A. Fractal geometry: what is it, and what does it do? [C]//Proceedings of the Royal Society of London A:

Mathematical, Physical and Engineering Sciences. The Royal Society, 1989, 423(1864): 3-16.

Marceau, D.J. (2005) Exploration of spatial scale sensitivity in geographic cellular automata. Environment and Planning B: Planning and Design 32, 693-714.

Ministry of Housing and Urban-rural

Development of the People's Republic of China (MOHURD), 2013. Chinese City Construction Statistics Yearbook 2012 (Beijing: China Planning Press).

Nation Bureau of Statistics, P. (1982) China's main demographic indicators from the 3rd national population census.

Nation Bureau of Statistics, P. (2011) China's main demographic indicators from the 6th national population census.

Reichman, O.J., M.B. Jones and M.P.

Schildhauer (2011) Challenges and opportunities of open data in ecology. Science 331.

Seto, K.C., M. Fragkias, B. Güneralp and M.K. Reilly (2011) A meta-analysis of global urban land expansion. PloS one 6, e23777.

Van de Voorde, T., W. Jacquet and F. Canters (2011) Mapping form and function in urban areas: An approach based on urban metrics and continuous impervious surface data. Landscape and Urban Planning 102, 143-55.

Wallace, J.L. (2014) Cities and Stability: Urbanization, Redistribution, and Regime Survival in China. Oxford University Press. Wei, Y. D. (2015). Spatiality of regional inequality. Applied Geography, 61, 1-10. Wei, Y. D., \& Ye, X. (2014a). Urbanization, land use, and sustainable development in China. Stochastic Environmental Research and Risk Assessment, 28(4), 755.

Wei, Y. D., \& Ye, X. (2014b). Urbanization, urban land expansion and environmental change in China. Stochastic environmental research and risk assessment, 28(4), 757-765. 